\title{
The energy source of the most energetic giant outbursts in MS $0735+7421$
}

\author{
Shuang-Liang Li \\ Key Laboratory for Research in Galaxies and Cosmology, Shanghai Astronomical Observatory, \\ Chinese Academy of Sciences, 80 Nandan Road, Shanghai 200030, China; \\ email: lisl@shao.ac.cn
}

\begin{abstract}
In this work, we investigate the power source of the most energetic giant outbursts in MS $0735+7421$, which released $\sim 10^{62}$ erg of energy. Due to the very high mean jet power in the cavities $\left(P_{\text {jet }} / L_{\mathrm{Edd}} \sim 0.02\right)$, we produce several jet formation models based on a relativistic thin disk model, i.e., general $B P+B Z$ mechanisms (model A), Livio's (model B) and Meier's (model $\mathrm{C}$ ) model, to explain the giant outbursts in AGNs. It is found that the energy provided by both model $\mathrm{B}$ and model $\mathrm{C}$ are inadequate for an initial black hole spin $a_{0} \sim 0.1$, only model A can explain the most violent outbursts in MS 0735+7421. But if the initial black hole spin $a_{0} \sim 0.95$, model B can also blow up the cavity. The final spin of the black hole is found to be very high in spite of the initial spin.
\end{abstract}

Keywords. Accretion, accretion disks, galaxies: active, black hole physics, quasars: general.

\section{Introduction}

The giant cavities in MS $0735+7421$, the most giant outburst so far, are found to contain an energy of $\sim 10^{62}$ erg (McNamara et al. 2009). These cavities are believed to be inflated by the jets launched from the active galactic nucleus (AGNs). There are several models to explain the formation of jets basing on the large-scale magnetic fields threading on the accretion disks and the ergosphere of the black holes, i.e., the famous Blandford-Znajek (BZ) mechanism extracting the rotational energies of the black holes Blandford \& Znajek (1977), Blandford-Payne (BP) mechanism extracting the rotational energies of the accretion disks (Blandford \& Payne 1982) and the hybrid model combining both the BZ mechanism and BP mechanism (Meier 1999).

In MS $0735+7421$, the mean jet power is $\sim 10^{46} \mathrm{erg} \mathrm{s}^{-1}$, which is about $0.2 \%$ of the Eddington luminosity. In this work, we explore the power source of the giant outbursts MS 0735+7421 and constraint on various popular models of jet formation and calculate the energy of jets by including both the BP process and the BZ process.

\section{Models}

The accretion rate during the outbursts is assumed to be high enough so the accretion flow will be a standard thin disk. The basic equations of a relativistic thin disk with magnetically driven outflows/jets includes: the continuity equation; the momentum equation; the angular momentum equation and the energy equation (see Li \& Cao 2012 for details). Moreover, we consider the evolution of Kerr black holes by accretion in AGNs as suggested by Moderski \& Sikora (1996). The jet power is calculated with three models, i.e., model A (general BZ +BP mechanisms, see Li \& Cao 2012 for details); model B (Livio's model, Livio et al. 1999, see Li \& Cao 2012 for details); model C (Meier's model, Meier 1999). 


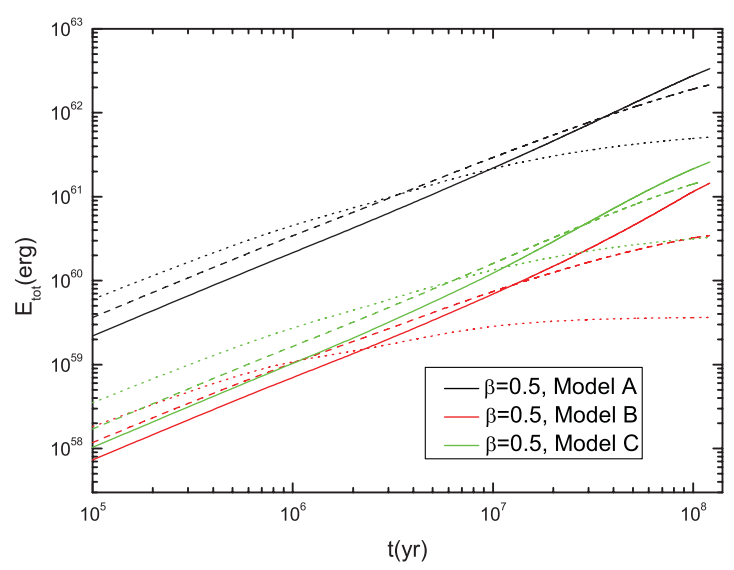

Figure 1. The energy released during the outbursts as functions of outburst time with $a_{0}=0.1$ and $\beta=0.5$. The black, red and green lines are calculated from model $\mathrm{A}, \mathrm{B}$ and $\mathrm{C}$ respectively. The solid, dashed and dotted line are for $\tau_{\mathrm{Q}}=10^{8}$ years, $\tau_{\mathrm{Q}}=5 \times 10^{7}$ years and $\tau_{\mathrm{Q}}=10^{7}$ years respectively.

\section{Results}

The evolution of mass accretion rate $\dot{M}$ can be calculated with the initial accretion rate $\dot{M}_{0}$ as suggested by Hopkins \& Hernquist 2009 (see Li \& Cao 2012 for details).

We compare the results of various models in Fig. 1 with the initial black hole spin $a_{0}=$ 0.1 for different quasar lifetime $\tau_{\mathrm{Q}}$. The jet accelerated from $B P$ and $B Z$ mechanisms in model A can provide enough energy to inflate the cavities in MS $0735+7421$. For model B (Livio's model), the jet power accelerated from accretion disk $L_{\mathrm{bp}}^{\text {livio }} \sim L_{\mathrm{bp}}(H / r)^{2}$ (the same for $B Z$ mechanism), which is far more smaller than that required by the cavities. The energy calculated by model C (Meier's model) is inadequate either, where the jet power is estimated at the inner stable orbit. But if the initial black hole spin $a_{0} \sim 0.95$, model B can blow up the cavity successfully. The final spin of the black hole is found to be very high in spite of the initial spin.

\section{Acknowledgements}

This work is supported by the NSFC (grants 10903021) and the Science and Technology Commission of Shanghai Municipality (10XD1405000).

\section{References}

Blandford, R. D. \& Payne, D. G. 1982, MNRAS, 199, 883

Blandford, R. D. \& Znajek, R. 1977, MNRAS, 179, 433

Hopkins, P. F. \& Hernquist, L. 2009, ApJ, 698, 1550

Li, S.-L. \& Cao, X. 2012, ApJ, 753, 24

Livio, M., Ogilvie, G. I., \& Pringle, J. E. 1999, ApJ, 512, 100

McNamara, B. R., Kazemzadeh, F., Rafferty, D. A., Birzan, L., Nulsen, P. E. J., Kirkpatrick, C. C., \& Wise, M. W. 2009, ApJ, 698, 594

Meier, D. L. 1999, ApJ, 522, 753

Moderski, R. \& Sikora, M. 1996, MNRAS, 283, 854 